\title{
Right Heart Thrombus in an Adult COVID-19 Patient: A Case Report
}

\author{
Enrico Merlo, Giuseppe Grutta, Ivo Tiberio, Gabriele Martelli* \\ Intensive Care Unit "U.O.C. Anestesia e Rianimazione" Department of Medicine (DIMED) Padua University Hospital, \\ Padua, Italy.
}

\begin{abstract}
Introduction: Right heart thrombus (RiHTh) can be considered a rare and severe condition associated with thromboembolic phenomena. A case is described of a COVID-19 patient presenting with an isolated thrombus in the right ventricle. Case presentation: An 80-years-old Caucasian male was admitted in an intensive care unit (ICU) for COVID-19 related acute respiratory distress syndrome. The patient showed signs of hemodynamic instability, elevated cardiac troponin I and altered coagulation. On further assessment, a thrombotic mass near the apex of the right ventricle was detected. Moreover, the apex and the anteroseptal wall of the right ventricle appeared akinetic. Following the administration of a therapeutic dose of unfractionated heparin over a forty-eight hour period, re-evaluation of the right chambers showed that the thrombotic mass had resolved entirely. Conclusion: COVID-19 patients could constitute a population at risk of RiHTh. Routine use of echocardiography and a multidisciplinary approach can improve the management of this condition.
\end{abstract}

Keywords: COVID-19, right heart thrombus, coagulation, echocardiography

Received: 23 May 2020 / Accepted: 21 October 2020

\section{INTRODUCTION}

Right heart thrombus is a life-threatening condition characterised by high early mortality due to pulmonary embolism [1].

Thrombi rarely originate in the right ventricle, in most cases, migrating there from the venous system as part of the thromboembolic phenomena [2].

However, the origin of a thrombus at the ventricular level may be established by the combination of hypercoagulability, blood stasis and intravascular vessel wall damage, the so-called Virchow's triad.

COVID-19 may lead to disseminated intravascular coagulation through the hyper-inflammation pathway.

Following the COVID-19 outbreak, several reports of patients with similar disorders have been published [3].

A recent study in which whole blood from patients admitted to an intensive care unit because of COVID-19, evaluated by thromboelastography, the TEG point-of-care device, concluded that the coagulation pattern might be linked to a state to hypercoagulability together with a severe inflammatory state [4].

Another critical factor is the cardiovascular damage associated with COVID-19. [5]
Guo et al. (2020) have shown that a significant correlation exists between inflammatory and myocardiocytolysis indices and a high incidence of cardiovascular complications, in COVID-19 hospitalised patients [6].

The pathophysiological mechanism of myocardial injury is still unclear, but it is hypothesised that hypoxia, hemodynamic instability, systemic inflammation and micro-thrombosis may be causative events. [5,7]

It should also be noted that the population most exposed to severe forms of COVID-19 presents a higher prevalence of cardiovascular and metabolic comorbidities, increasing the incidence of myocardial ischemia [7].

These factors suggest that the COVID-19 population may be at augmented risk for RiHTh.

\section{CASE PRESENTATION}

An 80-year-old Caucasian male, presenting with a high fever, was first admitted to the medical ward of the University Hospital of Padua, Italy, on the March 23rd 2020. Combined nasopharyngeal and oropharyngeal swabs were taken and tested positive for SARS-CoV-2. Three days after admission, on the March 26th, 2020, he was transferred to the ICU department of the hos- 
pital due to hypoxemic respiratory failure and a deterioration of his general condition. He was diagnosed as having acute respiratory distress syndrome (ARDS) related to COVID-19 disease.

His past medical history indicated that he had wellcontrolled systemic hypertension, treated with angiotensin-converting enzyme inhibitor, prostatic hypertrophy and early-stage cognitive dysfunction.

Upon transfer to the ICU, oxygen mask therapy was initiated plus $200 \mathrm{mg}$ hydroxychloroquine (Plaquenil ${ }^{\circledR}$, Sanofi, France) orally twice a day together with the antimicrobials, $500 \mathrm{mg}$ per day intravenous azithromycin (Zitromax ${ }^{\oplus}$, Pfizer, USA) and 2 grams per day intravenous ceftriaxone (Cefrtriaxone, Mylan Generics, Netherlands)

The patient was also administered 4000 UI enoxaparin (Inhixa, Techdow Pharma, Netherlands) subcutaneously, once a day and fitted with graded compression stockings as thrombosis prophylaxis.

On arrival at the ICU, the patient appeared agitated and dyspnoeic. The arterial blood gas analysis showed hypoxemic respiratory failure $(\mathrm{PaO} 256 \mathrm{mmHg}, \mathrm{PaCO} 2$ $35 \mathrm{mmHg}$ ), despite oxygen therapy delivered by a reservoir mask with an oxygen flow of $15 \mathrm{l} / \mathrm{min}$ ).

Immediately, respiratory support was commenced proceeding with orotracheal intubation and early mandatory ventilation. The ventilation was set in pressure control mode with pressure control $14 \mathrm{cmH}_{2} \mathrm{O}$, PEEP $10 \mathrm{cmH}_{2} \mathrm{O}$, tidal volume $7 \mathrm{ml} / \mathrm{kg}$, respiratory rate $16 /$ $\mathrm{min}, \mathrm{FiO}_{2}$ 0.7, and a static compliance of $51 \mathrm{ml} / \mathrm{cmH}_{2} \mathrm{O}$ and a $\mathrm{PaO} 2 / \mathrm{FiO}_{2}$ ratio of $110 \mathrm{mmHg}$ was recorded.

Laboratory test results showed a sudden elevation of troponin I (6700 ng/L), increased C-reactive protein $(100 \mathrm{mg} / \mathrm{L})$, altered haemostasis with INR 1.36, aPTT ratio 1.01 , fibrinogen $6.59 \mathrm{~g} / \mathrm{L}$, antithrombin III $71 \%$, and D-dimer $1168 \mu \mathrm{g} / \mathrm{L}$. Arterial-blood gases, after intubation, were recorded as $\mathrm{pH} 7.43 ; \mathrm{PaCO} 234 \mathrm{mmHg}$; $\mathrm{PaO} 273 \mathrm{mmHg}, \mathrm{HCO} 3-21.9 \mathrm{mmol} / \mathrm{L}, \mathrm{BE}-2.5 \mathrm{mmol} / \mathrm{L}$, $\mathrm{Hb} 13.1 \mathrm{~g} / \mathrm{dL} \mathrm{SaO} 295.5 \%$, lactate $1.6 \mathrm{mmol} / \mathrm{L}$.

In the early stages after the transfer to ICU, on the 3 rd day of stay, the patient showed hemodynamic instability that required norepinephrine (Noradrenalina, Galenica Senese, Italy) intravenous continuous infusion at a dose of $0,05 \mathrm{mcg} / \mathrm{kg} / \mathrm{mine}$ and dobutamine (Miozac, Fisiopharma, Italy) intravenous continuous infusion at a dose of $2 \mathrm{mcg} / \mathrm{kg} / \mathrm{min}$. A restrictive approach was chosen for fluid administration.

On the 3rd day of stay, an ECG 12-lead showed sinusal tachycardia with a recorded heart rate of 110 bpm and left anterior fascicular block. No previous ECGs were available for comparison, but there was no evidence of left anterior fascicular block in the patient's clinical history. The norepinephrine infusion was increased to $0,15 \mathrm{microgram} / \mathrm{kg} /$ minute overnight on the 3rd day of stay to treat the persistent hypotension.

Troponin-I levels were monitored throughout the whole length of stay. On the 3rd day of stay, a transitory elevation of troponin-I levels was recorded, but a significant decrease occurred in the following days.

A decision was taken to perform transthoracic echocardiography (TTE) on the fourth day of stay, and normal biventricular function with no signs of ventricular masses was noted.

On the 5th day of stay, a paroxysmal supraventricular tachycardia with hemodynamic impairment was reported. Following this event, we performed a fluidchallenge. The fluid-challenge only led to a temporary improvement in blood pressure parameters.

A MostCare Up (Vygon) monitoring system was employed to assess hemodynamic variables, and we found a low cardiac index $(2.1 \mathrm{l} / \mathrm{min} \cdot \mathrm{m} 2)$, normal stroke volume variation (11\%) and high systemic vascular resistance (2450 dyne.sec.m $2 / \mathrm{cm} 5$ ).

After an evaluation of the hemodynamic parameters, a decision was taken to increase the dobutamine infusion to $4,6 \mathrm{mcg} / \mathrm{kg} / \mathrm{min}$ and decrease the norepinephrine infusion to $0,05 \mathrm{mcg} / \mathrm{kg} / \mathrm{min}$.

The patient showed signs of haemodynamic improvement from the 6th to the 7th day of stay; the infusion of dobutamine and norepinephrine was progressively decreased and suspended on the 7 th day.

On 10th day of stay, a routine bedside TTE was performed resulting in the finding of a hyperechoic, pedunculated and mobile mass, about $9 \mathrm{~mm}$ in width and $16 \mathrm{~mm}$ in length, with a narrow implant base at the level of the apex of the right ventricle. The structure was attached to the interventricular septum (Figures 1-3).

After the acquisition of the echocardiographic images, an evaluation showed that the right ventricle presented an overall preserved systolic function with normal values of tricuspid annular plane systolic excursion (TAPSE) and regional alterations including apical and anteroseptal akinesia (Figure 4). The left ventricle showed a normal systolic function and a preserved ejection fraction of $53 \%$.

On the 10th day of stay, the patient's troponin I level was143 ng/L, a platelet count of 49410 9/L, INR 1.13, 
Available online at: www.jccm.ro

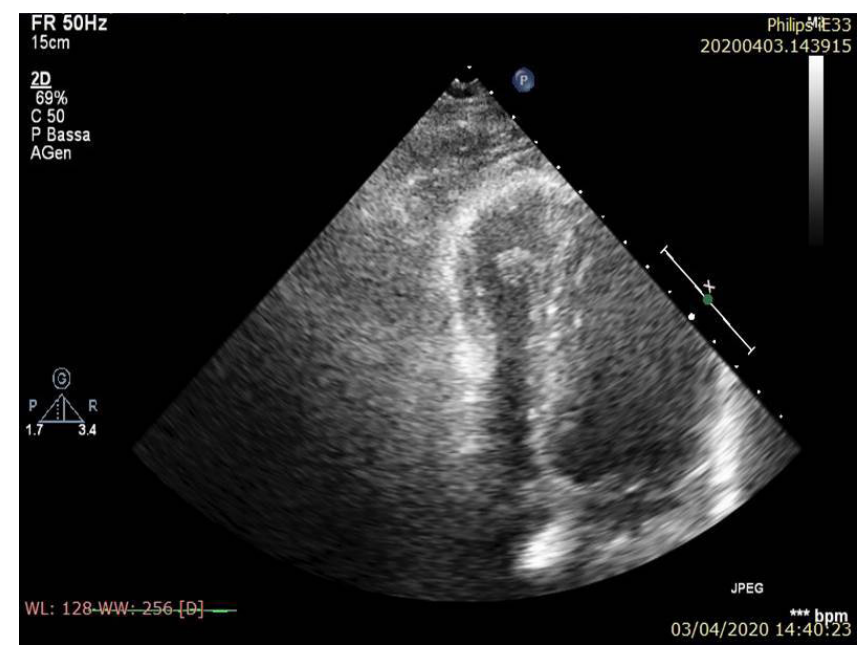

Fig.1. Intracardiac thrombus. TTE apical view. RiHTh as pedunculated, mobile mass of $9 \times 16 \mathrm{~mm}$ length, attached to the interventricular septum.

aPTT ratio 0.95 , fibrinogen $7.1 \mathrm{~g} / \mathrm{L}$, antithrombin III $104 \%$ and a rise in D-dimer value $(2655 \mu \mathrm{g} / \mathrm{L})$.

On the 11th day of stay, a consultant cardiologist confirmed the diagnosis of intracardiac thrombus and highlighted the presence of akinetic areas at the level of
The Journal of Critical Care Medicine 2020;6(4) • 239

the interventricular septum and the apex of the right ventricle. The cardiologist considered a previous ischemic event was likely based on the laboratory tests, the ECG indicating left anterior fascicular block, and the TTE findings.

In order to exclude other sites of thrombosis, systematic vascular US-assessment of upper and lower extremities was undertaken on 11th day of stay. No signs of systemic thrombosis were detected. The patient's Wells score was 6 points, indicating he was only at moderate risk of developing pulmonary embolism.

The medical team mutually agreed to start therapeutic anticoagulation with unfractionated heparin (Epsoclar, Pfizer, USA), with a targeted activated coagulation time (ACT) of 170-190 seconds.

Furthermore, from the 11th day of stay, $100 \mathrm{mg}$ oral acetylsalicylic acid (Cardioaspirin ${ }^{\oplus}$, Bayer, Germany) once a day as a prophylaxis and 2,5 mg beta-blocking agent bisoprolol (Cardicor, Recordati, Italy) orally once a day was started.

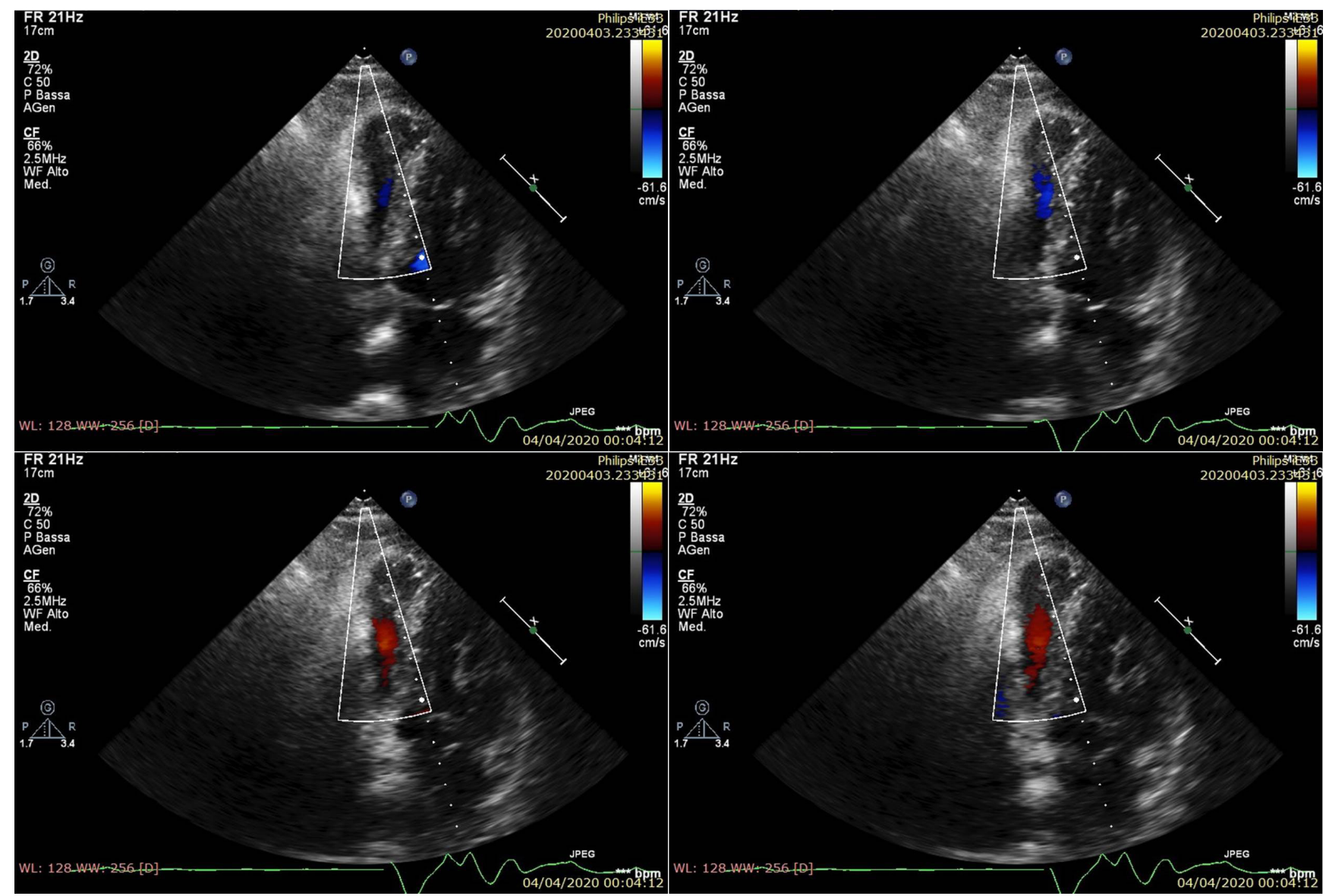

Fig. 2. Right ventricular flow pattern and thrombus mobility. TTE apical view, B-Mode/Color-Flow Mode. The systo-dyastolic motion of RiHTh alongside right wall contraction and its relationship with the intracardiac flow. 


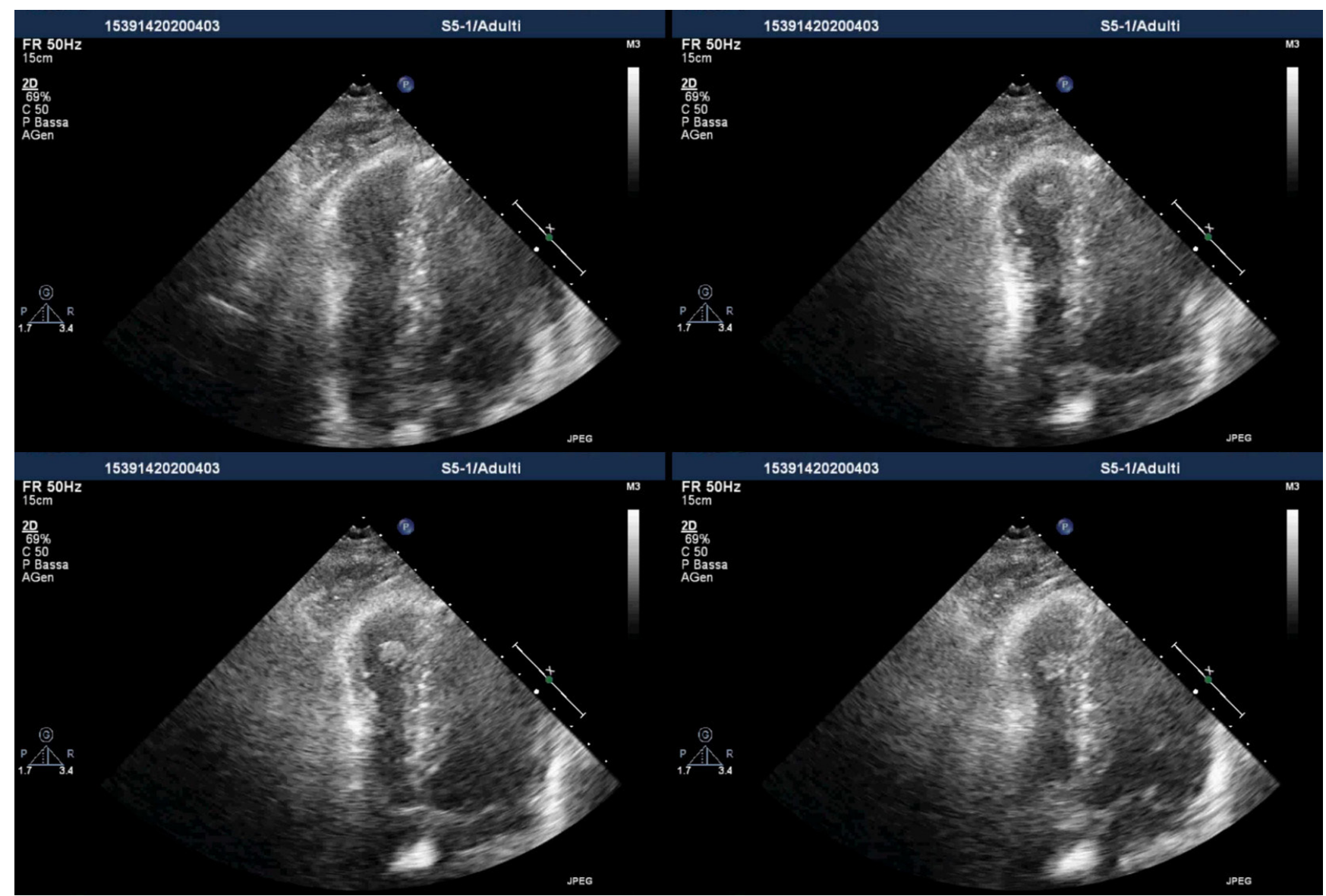

Fig. 3. Motion of the intracardiac thrombus. TTE apical view. Different positions of the thrombus during the cardiac cycle.

Following anticoagulation therapy, a steady decrease of D-dimer to a level of $936 \mu \mathrm{g} / \mathrm{L}$, was observed between the 11th and 21 st day of stay.

A reassessment carried out on the 14th day of stay. TTE images did not show the thrombus and confirmed the akinesia of the apical and anteroseptal portion of

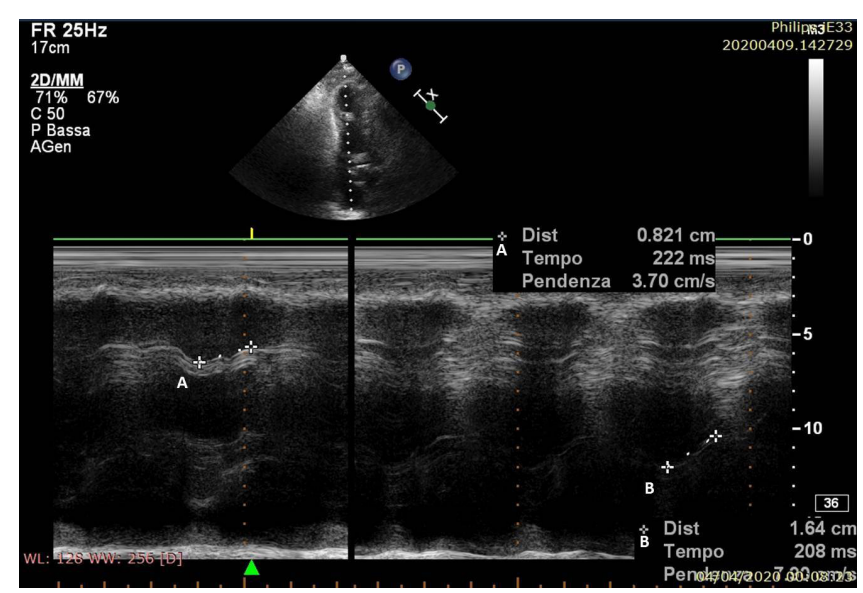

Fig. 4. M-MODE of the RiHTh. TTE apical view, M-Mode. The excursion of the RiHTh $(A, 8.21 \mathrm{~mm})$, and tricuspid annular plane (B, TAPSE $16.4 \mathrm{~mm}$ ). the right ventricle. The thrombus had resolved without hemodynamic alterations or worsening of respiratory exchanges.

These findings led to the case being re-evaluated; low-molecular-weight heparin (Inhixa, Techdow Pharma, Netherlands) was reintroduced with the integration of anti-Xa activity monitoring with a targeted level of $0,5-1 \mathrm{kU} / \mathrm{L}$.

This was continued until the patient was discharged from the ICU. On the 17th day of stay, the patient was extubated, and after thirty-six days was transferred to a long-term care unit.

\section{DISCUSSION}

This report is a description of a rare complication, a right heart thrombus, occurring in a patient with COVID-19 related ARDS.

RiHTh aetiology was not related to a deep vein thrombosis or vascular catheter, unlike other reported cases of COVID-19 patients $[8,9]$. The morphology of 
the thrombus and patient's clinical history suggested a multifactorial aetiology.

A type C floating thrombus was recorded and defined as such according to the classification of the European Working Group on Echocardiography [10].

In our opinion, the "in situ" development could be a consequence of recent myocardial ischemia and its occurrence facilitated by the presence of akinetic segments of the right ventricle. As laboratory tests confirmed the diagnosis of hypercoagulability, COVID-19 related hypercoagulability could have been fundamental for its growth [11].

The diagnosis of hypercoagulability was corroborated by high serum values of $\mathrm{D}$-dimer and fibrinogen, parameters which have been identified as indices that reflect hypercoagulable status in COVID-19 patient [12].

Early performed echocardiography was useful in identifying the RiHTh and allowed to set therapy with good timing, avoiding unnecessary examinations and further complications. Routine use of echocardiography should be considered in COVID-19 patients at risk of pulmonary embolism.

The patient was treated with anticoagulants at therapeutic dose after a multidisciplinary discussion of the case. The optimal treatment for similar clinical situations is debatable. Analysing the impact on mortality between fibrinolytic treatment and anticoagulation in patients with RiHTh, there is no evidence of the superiority of one treatment over the other [13]. Considering the frequent association of RiHTh with pulmonary embolism, fibrinolytic treatment may lead to outcome improvement in some cases, especially when the right ventricular dysfunction is present [14]. In our case, the function of the right ventricle appeared preserved despite the presence of regional alterations. Consequently, the anticoagulant treatment with unfractionated heparin seemed the most suitable treatment considering the clinical condition and the bleeding-risk of the patient.

\section{CONCLUSION}

RiHTh could represent an emerging complication in COVID-19 patients and deserves the utmost attention given the high associated mortality. We suspect that hypercoagulability and myocardial damage could represent a causative pattern of RiHTh in COVID-19, but further studies are needed to confirm this hypothesis.
The diagnosis and treatment of RiHTh can be challenging. In the first place, it is essential to recognise whether predisposing factors are present and if the patient is at risk of developing RiHTh. Furthermore, we think that routine echocardiographic exams in selected patients and a multidisciplinary approach could improve the diagnostic and therapeutic process.

\section{ETHICS APPROVAL AND CONSENT}

Ethics approval and consent to participate: the authors state that this study was performed following the ethical principles of the World Medical Association's Declaration of Helsinki. Written informed consent was obtained from the patient for publication of this case and any accompanying images.

\section{- CONFLICT OF INTEREST}

The authors have no competing interests.

\section{DEFERENCES}

1. Konstantinides SV, Meyer G, Becattini C, et al. 2019 ESC Guidelines for the diagnosis and management of acute pulmonary embolism developed in collaboration with the European Respiratory Society (ERS): The Task Force for the diagnosis and management of acute pulmonary embolism of the European Society of Cardiology (ESC). Eur Respir J. 2019;54(3):1901647. doi:10.1183/13993003.01647-2019

2. Koć $M$, Kostrubiec M, Elikowski W, et al. Outcome of patients with right heart thrombi: the Right Heart Thrombi European Registry. Eur Respir J. 2016;47(3):869-875.

3. Tang N, Li D, Wang X, Sun Z. Abnormal coagulation parameters are associated with poor prognosis in patients with novel coronavirus pneumonia. J Thromb Haemost. 2020;18(4):844847.

4. Panigada M, Bottino N, Tagliabue $P$, et al. Hypercoagulability of COVID-19 patients in Intensive Care Unit. A Report of Thromboelastography Findings and other Parameters of Hemostasis. J Thromb Haemost. 2020;18(7):1738-1742.

5. Guzik TJ, Mohiddin SA, Dimarco A, et al. COVID-19 and the cardiovascular system: implications for risk assessment, diagnosis, and treatment options. Cardiovasc Res. 2020; 116(10):1666-87.

6. Guo T, Fan Y, Chen M, et al. Cardiovascular Implications of Fatal Outcomes of Patients With Coronavirus Disease 2019 (COVID-19). JAMA Cardiol. 2020; 5(7):1-8.

7. Li B, Yang J, Zhao F, et al. Prevalence and impact of cardiovascular metabolic diseases on COVID-19 in China. Clin Res Cardiol. 2020;109(5):531-538. 
242 - The Journal of Critical Care Medicine 2020;6(4)

8. Elkattawy S, Younes I, Noori MAM. A Case Report of Polymerase Chain Reaction-Confirmed COVID-19 in a Patient With Right Ventricular Thrombus and Bilateral Deep Vein Thrombosis. Cureus. 2020;12(6):e8633

9. $\mathrm{Hu} \mathrm{D}$, Liu K, Li B, Hu Z. Large intracardiac thrombus in a COVID-19 patient treated with prolonged extracorporeal membrane oxygenation implantation. European Heart Journal. 2020;41(32):3104-3105.

10. THE EUROPEAN WORKING GROUP ON ECHOCARDIOGRAPHY, Kronik G. The European Cooperative Study on the clinical significance of right heart thrombi. Eur Heart J. 1989;10(12):1046-1059.

11. Sulemane S, Baltabaeva A, Barron AJ, Chester R, Rahman-Haley
Available online at: www.jccm.ro

S. Acute pulmonary embolism in conjunction with intramural right ventricular thrombus in a SARS-CoV-2-positive patient. Eur Heart J Cardiovasc Imaging. 2020;21(9):1054.

12. Joly BS, Siguret V, Veyradier A. Understanding pathophysiology of hemostasis disorders in critically ill patients with COVID-19. Intensive Care Med. 2020;46(8):1603-1606.

13. Barrios D, Chavant J, Jiménez D, et al. Treatment of Right Heart Thrombi Associated with Acute Pulmonary Embolism. Am J Med. 2017;130(5):588-595.

14. Meyer G, Vicaut E, Danays T, et al. Fibrinolysis for Patients with Intermediate-Risk Pulmonary Embolism. N Engl J Med. 2014;370(15):1402-1411. 